

to a close spiral (Fig. $A$ ), and from a lower temperature a coarser spiral. A temperature gradient along the tube, made by touching one end of the tube to the hot plate and raising the other end slightly, end of the tube to the hot plate and raising the other end slightly,
results in a tapered spiral (Fig. $B$ ). Too low temperature of heating results in a tapered spiral (Fig. $B$ ). Too low temperature of heating
before quenching gives a straight line or a wavy line fracture, while a too high temperature may cause short segments of the tube to be broken off. Left- and right-handed spirals are equally prevalent, even in sections of the same tube. in sections of the same tube. describe the cracking of the glass as follows. Well-annealed glass is strain-free when uniformly heated. When such hot glass is suddenly plunged for a moment into water, the outside of the glass in contac inth the water is chilled and undergoes tensional stress while the hot interior is compressed. Under the tensional stress, any minute flssure in the surface of the glass (ustally at the end) may open and start a crack which travels over the surface of the glass and relieves the tensional strain. Prolonged cooling deepens the crack and causes a secondary crack to form which is continuous with the first and com-
pletes the spital rupture. The spiral pattern is apparently the one that gives most strain release in the circumstances.

The simplicity of this form of fracture would indicate that the related mathematical problem in heat transfer and stress-strain relations might have a relatively simple solution.

Applied Physics Laboratory,

Johns Hopkins University,

Silver Spring, Maryland. Sept. 22.

* Contribution No. 4 from the Applied Physics Laboratory of The Johns Hopkins University ; the work described here was done in Johns Hopkins University; the work described here was done in
connexion with Contract NOrd 7386 with the U.S. Naval Bureau of connexion
Ordnance.

\section{Electron Accelerator of Synchrotron Type}

WHuLE accelerators of the cyclotron type have facilitated the production of energetic particles, they are, as yet, beyond the financial means of a great many laboratories. Moreover, the attainment of energies nearing the range of a thousand million electron-volts is current methods. It is suggested that, by using the synchrotron current methods. It is suggested that, by using the synchrotron
principle $^{1}$ together with a magnet of unusual design, these objections principle ${ }^{x}$ together wight be overcome.
math

It can be shown that the energy and radius of an equilibrium orbit in the synchrotron are determined by

$$
\begin{aligned}
& E=\sqrt{(B c e r)^{2}+E_{0}{ }^{2}}, \\
& r=\sqrt{(c / \omega)^{2}-\left(E_{0} / B c e\right)^{2}}
\end{aligned}
$$

where $E$ is equilibrium energy (total); $E_{0}$ is rest mass energy ; $r$ is radius of equilibrium orbit ; $B$ is magnetic flux density at orbit; $\omega$ is angular velocity of 'dee' voltage ; $e$ is charge on particle ; $c$ is velocity of light.

Equation (1) shows that the equilibrium energy may be increased by increasing $B$-as observed by McMillan. Equation (2) indicates that the equilibrium radius may be maintained constant by causing a suitable increase in $\omega$ as the value of $B$ is raised.

Rendering the equilibrium radius constant in this way allows the use of a magnet of simplified design. The most convenient form of use of a magnet of simplified design. The most convenient form of
magnet is a laminated steel bobbin, the depth and width of which are small compared with its diameter. The vacuum chamber and are small compared with its diameter. The vacuum chamber and
energizing coil lie between the cheeks of the bobbin, the coil having energizing coil lie between the cheeks of the bobbin, the coil having
the smaller diameter. Ring-shaped pole pieces are fastened to the the smaller diameter. Ring-shaped pole pieces are fastened to the
cheeks in the region of the vacuum chamber so that the distribution cheeks in the region of the vacuum chamber so that the distribution
of the field may be controlled. The usual magnet yoke is eliminated in this way and, since it is unnecessary to increase the depth and width of the bobbin in direct proportion to the diameter, the saving in material and the efficiency are greater for larger accelerators. The magnet of the small (13 Mev.) electron accelerator which is being The magnet of the small ( 13 Mev.) electron acce
built at this University weighs less than 200 lb.

While some difficulty is associated with the required change in $\omega$, no insuperable difficulty is anticipated. It is certainly possible to produce a change large enough to accelerate electrons to high energy from a reasonable injection energy. It is thought that, where the final velocity of an accelerated particle is several times the initial
value, the difficulty of producing a correspondingly large frequency change might be circumvented by the use of 'harmonic orbits'. The fact that a particle can be accelerated when its period is an integral multiple of the period of the 'dee' voltage suggests that a large change in velocity may be accommodated by repeatedly changing $\omega$ over a 2: 1 range. The frequency is increased slowly and decreased very rapidly, several such cycles occurring as the magnetic field increases to its maximum. We hope to verify this when our accelerator is placed in operation.

University of the Witwatersrand,

A. I. ARCHER Sept. 10 .

'McMillan, E. M., Phys. Rev., 68, 143 (1945).

Reaction Velocity at Phase Limits and its Dependence on the Frequency of the Vibration of the Lattice

IN studying reactions between two solid phases, it is found that the reaction velocity in systems such as $\mathrm{MgO} / \mathrm{Ag}_{2} \mathrm{SO}_{4}{ }^{1}, \mathrm{MgO}_{\mathrm{Ag}} \mathrm{PO}_{4}{ }^{1}$, $\mathrm{MgO} / \mathrm{Mg}_{2} \mathrm{P}_{2} \mathrm{O}_{7}{ }^{2}$ and $\mathrm{MgO} / \mathrm{MgSiO}_{3}{ }^{3}$ is not determined by the difrusion process through the reaction products, but by reactions at one of the phase limits. The reaction velocity is independent of temperature according to the exponential equation

$$
d m / d t=C \cdot \exp (-q / R T) .
$$

The reaction velocity of the systems magnesium oxide/silver salt is at a certain temperature about a million times as great as in the system $\mathrm{MgO} / \mathrm{Mg}_{2} \mathrm{P}_{2} \mathrm{O}_{7}$, and in the latter is considerably greater than in the system $\mathrm{MgO} / \mathrm{MgSiO}_{3}$; but this difference is dependent only on the great differences in energy of activation. On the other hand, the constant $C$ for all the four systems is practically the same.

$\begin{array}{ccc}\text { System } & q \text { kcal. } & C \text { gm.-mol. } \mathrm{MgO} \mathrm{cm} .^{-2} \mathrm{sec}^{-t} \\ \mathrm{MgO} / \mathrm{Ag}_{2} \mathrm{SO}_{4} & 61 & 2 \cdot 0 \times 10^{5} \\ \mathrm{MgO} / \mathrm{Ag}_{3} \mathrm{PO}_{3} & 61 & 2 \cdot 0 \times 10^{5} \\ \mathrm{MgO} / \mathrm{Mg}_{2} \mathrm{P}_{3} \mathrm{O}_{7} & 82 & 2 \cdot 1 \times 10^{5} \\ \mathrm{MgO} / \mathrm{MgSiO}_{3} & 112 & 1 \cdot 0 \times 10^{5}\end{array}$

Since the specific gravity of magnesium oxide is about $3 \cdot 2$, the constant $C$ corresponds to a yield of $5 \times 10^{13}-1.0 \times 10^{14}$ molecule layers per second : the linear reaction velocity in $\mathrm{cm}$. sec.-1 is in the systems investigated proportional to the product of atom frequency and lattice spacing of the oxide 4 .

When investigating the thermal decomposition of zinc oxide we have arrived at an analogous result ${ }^{5}$ :

$$
d x / d t=1.2 \times 10^{12} \exp (-94,000 / R T) .
$$

Department of Chemical Technology,
Chalmers College of Technology,

ROBERT JAGITSCH

$$
\begin{aligned}
& \text { rothenburg. } \\
& \text { Sept. } 2 \text {. }
\end{aligned}
$$

I Jagitsch, R., and Hedvall, J. A., Ark. kemi., min. o. geol. (Stockholm). 19 A, No. 14 (1944).

${ }^{2}$ Jagitsch, R., and Perlström, G., Ark. kemi., min. o. geol. (Stockholm), 22 A, No. $4(1946)$.

${ }^{3}$ Unpublished results.

4 cf. Polanyi, M., and Wigner, E., Z. phys. Chem., A, 139, 439 (1928).

Bengtson, B., and Jagitsch, R., Ark. kemi., min. o. geol. (Stockholm), in the press.

An Extension of the Lens-Mirror System of Maksutor

THE lens-mirror system described by D. D. Maksutov ${ }^{1}$, in which the aberrations of a spherical mirror are corrected by a single sphericalsurfaced menisens lens, while eminently suitable for telescope objectives of moderate relative aperture, combining the coma correction of the refractor with a virtual absence of secondary spectrum, suffers from two sets of limitations which restrict its possible application in other flelds. In the first place, the higher order spherical aberration is too great to yield the highest resolving power at very great relative apertures (except at very small focal lengths). Secondly, since the system has only three variables apart from meniscus thickness, the with spherical aberration and axial achromatism, thus restricting its with spherical aberration and axial achromatism, thus restricting its by increased thickness of the meniscus, but this necessarily involves by increased thickness of the meniscus

D. G. Hawkins and E. H. Linfoot ${ }^{2}$ recently described in Nature a combination of a concentric Maksutov meniscus and a doublet Schmidt aspheric plate which overcomes these limitations. Similar results may be obtained without the use of non-spherical curves by the use of be obtained without the use of non-spherical curves by the use of two spherical-surfaced meniscus lenses, one concave and one convex to
the mirror. In such a system, conserving the secondary-spectrum the mirror. In such a system, conserving the secondary-spectrum correction of the Maksutor system, the higher order spherical aberramay be completely corrected, and the meniscus thicknesses may be may be completely corrected, and the meniscus thicknesses may be detriment to the oblique imagery. Moreover, the variables of the detriment to the oblique imagery. Moreover, the variables of the system being more than are required to rulifl the Seidel conditions, a form of lens mav be chosen reducing the higher order oblique aberrations to negligible size; this is possible in these two-meniscus systems by adopting a form in which the effective stoplies between the centres of curvature of the two surfaces. of each meniscus (which are close together), thus reducing the angles of incidence of a principal ray to very small values. This is not possible in the Maksutov systems, tively far removed from the meniscus centres of curvature. 